\title{
Duodenopancreatectomia e hemicolectomia direita em monobloco para tratamento de câncer de cólon direito localmente avançado
}

\section{En bloc pancreaticoduodenectomy and right hemicolectomy for locally advanced right colon cancer treatment}

Alexandre Cruz Henriques, TCBC-SP; Jaques Waisberg, TCBC-SP1; Karla de Andrade Possendoro²; Felipe Emanuel Fuhro 3 ; Manlio Basílıo Speranzini, ECBC-SP4

\section{INTRODUÇÃO}

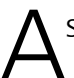
neoplasias malignas coloretais são muito comuns no Ocidente' ${ }^{1}$ O câncer de cólon localmente avançado é uma afecção freqüente, ocorre principalmente no sigmóide e reto, nestas localizações pode invadir diversas vísceras vizinhas, tais como útero, bexiga intestino delgado, entre outros $^{2}$. Nestes casos, a ressecção multivisceral está consagrada $^{3}$. Os tumores do cólon direito aderidos ao duodeno ou pâncreas representam um dilema para o cirurgião, devido à magnitude e a complexidade de uma duodenopancreatectomia associada a hemicolectomia direita ${ }^{3}$. O objetivo deste artigo é relatar um caso de câncer de cólon direito localmente avançado tratado com gastroduodenopancreatectomia cefálica associada a hemicolectomia direita em monobloco.
\end{abstract}

\section{RELATO DO CASO}

Paciente masculino, 63 anos, procurou assistência médica com quadro de diarréia, presença de alimentos nas fezes há um mês.Os episódios de diarréia ocorriam geralmente poucos minutos após a ingestão de alimentos e eram acompanhados por cólica abdominal. Apresentava também astenia e emagrecimento de $12 \mathrm{~kg}$ no período. $\mathrm{O}$ exame físico do abdome mostrava discreto plastrão mal definido, levemente doloroso a palpação profunda em hipocôndrio direito.

Com hipótese diagnóstica de fístula gastrocólica ou fístula jejunocólica, foram solicitados endoscopia digestiva alta e radiografia contrastada do trânsito de delgado. O primeiro revelou lesão ulcerovegetante na segunda porção duodenal, com subestenose local. O exame anátomopatológico revelou adenocarcinoma papilífero indiferenciado. A radiografia contrastada do trânsito intestinal mostrou fístula duodenocólica (Figura 1).

Tomografias computadorizadas de tórax, de abdome e pelve foram solicitadas para estadiamento e o único achado relevante foi o espessamento da parede abdominal do ângulo hepático do cólon; nenhum sinal de disseminação metastática foi observado. Avaliação clínica préoperatória era normal.

Com hipótese diagnóstica de tumor de origem colônica fistulizado para duodeno, o paciente foi submetido à laparotomia exploradora. Observou-se lesão neoplásica, no ângulo hepático do cólon, invadindo exten-

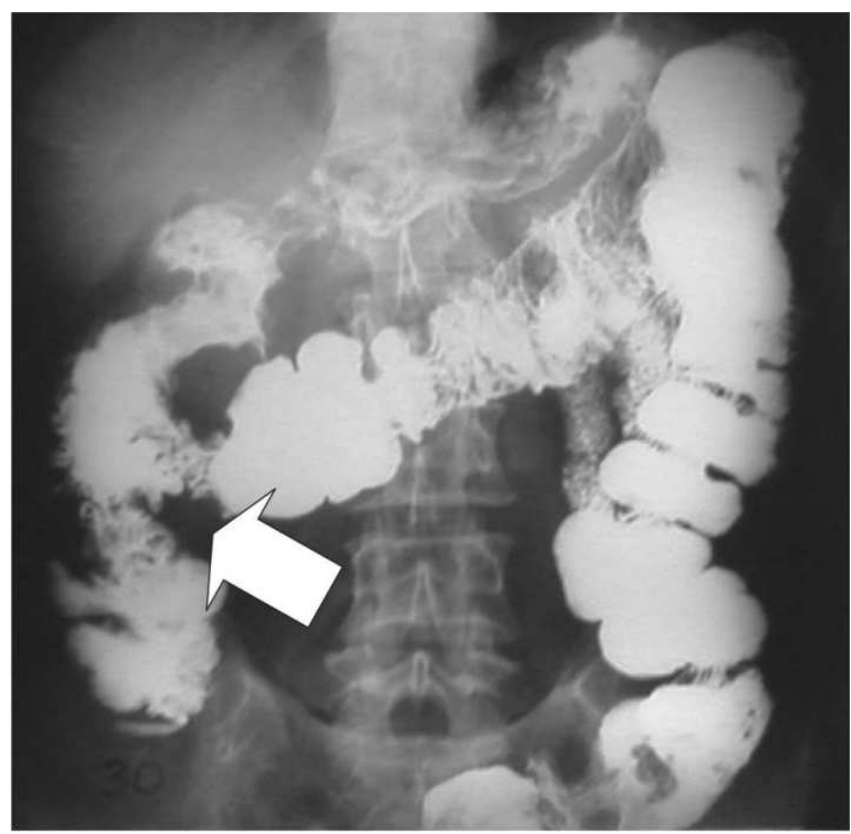

Figura 1 - Radiografia contrastada do trânsito intestinal mostrando fístula duodenocólica.

Trabalho realizado no Hospital de Ensino da Faculdade de Medicina do ABC- São Bernardo do Campo-SP-BR.

1. Professor Assistente da Disciplina de Cirurgia do Aparelho Digestivo da Faculdade de Medicina do ABC- São Bernardo do Campo-SP-BR; 2. Cirurgião do Pronto Socorro Central de São Bernardo, Faculdade de Medicina do ABC- São Bernardo do Campo-SP-BR; 3. Cirurgião do Hospital de Ensino da Faculdade de Medicina do ABC; 4. Professor Titular da Disciplina de Cirurgia do Aparelho Digestivo da Faculdade de Medicina do ABC- São Bernardo do Campo-SP-BR. 
samente segunda porção duodenal. Realizada gastroduodenopancreatectomia cefálica associada a hemicolectomia direita em monobloco (Figuras 2 e 3) reconstrução do trânsito com anastomose coledocojejunal, pancreatojejunal e gastrojejunal com alça única, e ileotransverso anastomose. (Figura 2)

O paciente evoluiu no $7^{\circ}$ dia de pós-operatório com evisceração. Foi submetido à ressutura da parede abdominal, e recebeu alta no $10^{\circ}$ dia de pós-operatório.

O estudo anátomo-patológico da peça mostrou "adenocarcinoma moderadamente diferenciado", do cóIon direito, infiltrando duodeno, cabeça do pâncreas e gordura peri-cólica. Margens de ressecção livres de doença, ausência de disseminação linfática (0/28). Painel imunohistoquímico demonstrou carcinoma tipo mucinoso compatível com primário em segmento colônico.

O serviço de oncologia consultado optou por não realizar tratamento adjuvante. Atualmente, 48 meses após a ressecção do tumor o paciente encontra-se sem sinais de disseminação ou recidiva da doença.

\section{DISCUSSÃO}

Os tumores localmente avançados são caracterizados por aderências neoplásicas em órgãos adjacentes, na ausência de metástases à distância'.

O câncer de cólon afeta 5\% da população e 10\% desses tumores envolverão estruturas adjacentes, principalmente aqueles situados no sigmóide e reto ${ }^{4}$. O câncer de cólon direito e transverso proximal apresenta-se aderido a órgãos adjacentes em $11 \%$ a $28 \%$ dos casos, e pode invadir o duodeno e cabeça de pâncreas².

Grey Turner em 1929 publicou a primeira ressecção duodenal para câncer de cólon localmente avançado, e Van Prohaska et al. realizaram a primeira duodenopancreatectomia para câncer de cólon direito avançado em $1953^{1}$.

Os pacientes com tumor de cólon direito localmente avançado apresentam quadro clínico muito variado, podendo ocorrer sangramento gastrointestinal, anorexia, emagrecimento e diarréia, esta última manifestação indicativa de fístula duodenocólica ${ }^{2,4}$.

Os tumores localmente avançados de cólon direito, podem ser suspeitados no pré-operatório através do achado de massa palpável no abdome ou quando a tomografia computadorizada de abdome, mostra massa hipodensa envolvendo órgão adjacentes ${ }^{1}$. Às vezes, não há sinais clínicos ou radiológicos de invasão tumoral de vísceras adjacentes, sendo esse um achado intra-operatório.

A diferenciação entre aderências inflamatórias ou neoplásicas, nem sempre pode ser realizada1. Sabe-se que $40 \%$ dos tumores aderidos em órgãos vizinhos têm aderências inflamatórias e não neoplásicas. Mesmo assim é mandatória a ressecção multivisceral, uma vez que, quan-

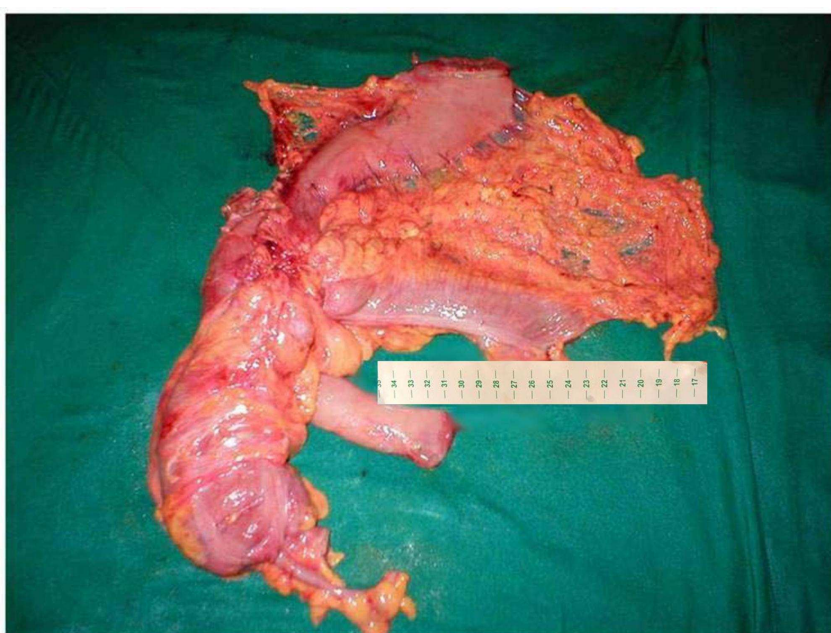

Figura 2 - Gastroduodenopancreatectomia cefálica associada a hemicolectomia direita em monobloco (posição anatômica).

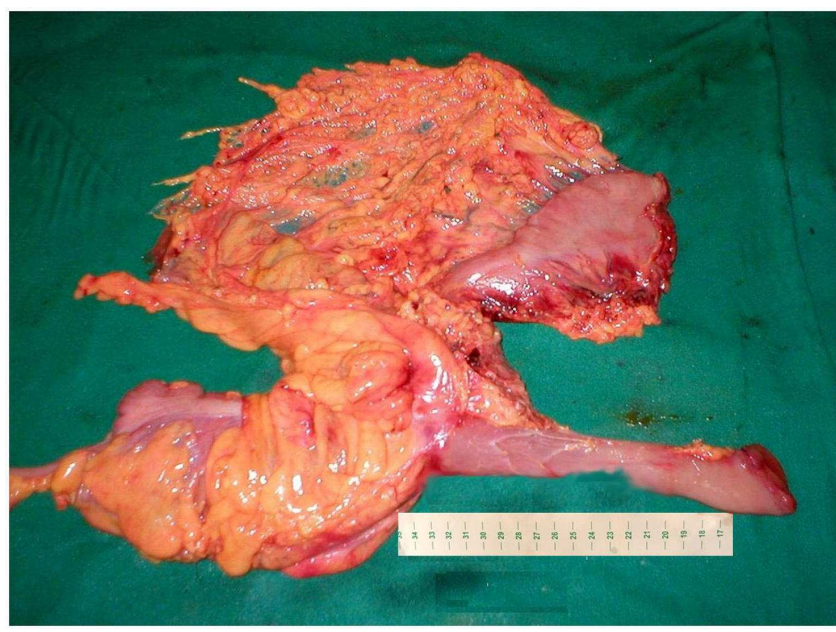

Figura 3 - Gastroduodenopancreatectomia cefálica associada a hemicolectomia direita em monobloco (colon rebatido).

do a aderência é neoplásica a separação do tumor de uma estrutura adjacente produz recorrência local em 100\% dos casos $^{5}$.

Antes de realizar uma ressecção multivisceral, a condição clínica do paciente e a presença ou não de metástases à distância devem ser consideradas. A taxa de mortalidade das ressecções em bloco é de $3 \%{ }^{3}$.

As ressecções multiviscerais em monobloco, associam-se com tempo de sobrevivência médio de 40 meses e taxa de sobrevivência em cinco anos livre de doença de $54 \%$. Já a sobrevivência média do "bypass" paliativo é de nove meses, e da ressecção incompleta, incluindo a ressecção com separação de órgãos é de 11 meses ${ }^{4}$.

O longo período de sobrevivência após ressecções multiviscerais, pode ser explicado pela ausência de metástases em linfonodos regionais (NO), em 45\% dos $\operatorname{casos}^{5}$. como no caso aqui apresentado, evidenciando um subgrupo biológico que, apesar de localmente agressivos, 
tem baixa incidência de disseminação linfonodal². A literatura consultada, mostra que se o paciente tiver condições clínicas adequadas e não apresentar metástases à distância, a ressecção multivisceral é o tratamento de escolha, mesmo quando a operação associada tem a magnitude e complexidade de uma duodenopancreatectomia ${ }^{1-5}$.
O papel da quimioterapia adjuvante no tratamento de tumores localmente invasivos é incerto ${ }^{4}$. A quimioterapia sistêmica é benéfica para pacientes com metástases linfonodais, mas restam controvérsias se esta oferece benefícios para pacientes com linfonodos negativos, mesmo em neoplasias no estágio pT3 ou pT44.

\section{A B S S T R A C T}

This article reports the case of a patient whit a diagnosis of diarrhea and weight loss. Subsidiary exams showed ulcerovegetant lesion in the second duodenal portion and duodenocolic fistula. An exploratory laparotomy was performed and a neoplasic lesion in the hepatic angle of the colon was observed invading the second duodenal portion. The patient then underwent a cephalic gastroduodenopancreatectomy associated with en bloc right hemicolectomy and improved well in the postoperative period. Currently, 48 months after the surgery, he does not present any signs of the disease dissemination or recurrence. The consulted literature recommends that multivisceral resection must be considered if the patient is clinically able to undergo major surgery and does not present any signs of neoplasic dissemination, since the postoperative survival time is considerably longer in the resected group and some of these patients even achieve cure.

Key words: Colorectal neoplasms. Pancreaticoduodenectomy. Colectomy.

\section{REFERÊNCIAS}

1. Perez RO, Coser RB, Kiss DR, Iwashita RA, Jukemura J, Cunha JE, Habr-Gama A. Combined resection of the duodenum and pancreas for locally advanced colon cancer. Curr Surg. 2005; 62(6):613-7.

2. Curley SA, Evans DB, Ames FC. Resection for cure carcinoma of the colon directly invading the duodenum or pancreatic head. J Am Coll Surg. 1994; 179(5): 587-92.

3. Gebhardt MW, Ruckriegel S. Multivisceral Resection of advanced colorectal carcinoma.Langenbecks Arch Surg. 1999; 384(2): 1949.

4. Koea JB, Conlon K, Paty PB, Guillem JG, Cohen AM. Pancreatic or duodenal resection or both for advanced carcinoma on the right colon: is it justified? Dis Colon Rectum. 2000; 43(4): 460-5.

5. Berrospi F, Celis J, Ruiz E, Payet E. En bloc pancreaticoduodenectomy for right colon cancer invading adjacent organs. J Surg Oncol. 2002; 79(3): 174-97; discussion 198
Recebido em 05/06/2006

Aceito para publicação em 20/08/2006

Conflito de interesse: nenhum

Fonte de financiamento: nenhuma

Como citar este artigo:

Henriques AC, Waisberg J, Possendoro KA, Fuhro FE, Speranzini MB. Duodenopancreatectomia e hemicolectomia direita em monobloco para tratamentode câncer de cólon direito localmente avançado. Rev Col Bras Cir. [periódico na Internet] 2010; 37(3). Disponível em URL: http://www.scielo.br/rcbc

Endereço para correspondência:

Alexandre Cruz Henriques

E-mail: achenriques@uol.com.br 